\title{
Necesidad de un marco de referencia para caracterizar el pensamiento físico: algunas reflexiones.
}

\section{Necessity of reference framework to characterize the physics thinking: some reflections.}

\author{
Marcos Campos-Nava ${ }^{\mathrm{a}, ~ * ~(D), ~ A g u s t i ́ n ~ A l f r e d o ~ T o r r e s-R o d r i ́ g u e z ~}{ }^{\mathrm{b}}$ ic
}

a Área Académica de Matemáticas y Física, Universidad Autónoma del Estado de Hidalgo, 42184, Pachuca, Hidalgo, México.

${ }^{b}$ Departamento de Ciencias Básicas, Tecnológico Nacional de México, Instituto Tecnológico de Atitalquia, 42970, Atitalaquia, Hidalgo, México.

\begin{abstract}
Resumen
Se presentan una serie de reflexiones con la intención de contribuir a consolidar un marco de referencia que puede permitir caracterizar algunos aspectos del pensamiento físico. La literatura en español e inclusive en inglés es escasa al respecto, si bien se habla de un pensamiento científico, un pensamiento crítico y hasta un pensamiento matemático, es difícil encontrar referencias respecto a ¿Qué debemos entender por pensamiento físico? ¿Qué significa pensar en términos de la física? Los autores consideran pertinente incluir tópicos como este en las investigaciones en didáctica de la física, puesto que la caracterización del pensamiento físico -en caso de que sea posible- podría aportar elementos que permitan entender ¿por qué resulta tan complicado para algunas personas entender la física? El presente trabajo que tiene fines divulgativos principalmente, es una revisión de la literatura respecto al tema, y tal cual se sugiere en el título, se busca fomentar la reflexión respecto al tema.
\end{abstract}

Palabras Clave:

Pensamiento Físico, Pensamiento Científico, Física Educativa.

\begin{abstract}
In this proposal we presented a set of reflections with the aim to contribute to consolidate one reference framework that allow characterize some aspects of physics thinking. Literature in Spanish, even in English about this topic is scarce, some researches talk about scientific thinking, critical thinking, even about mathematical thinking, nevertheless is complicate to find literature that explain, What is physics thinking? What means to think in terms of physics science? The authors consider pertinent to include such topics in the research of physics education, since the characterization of physical thinking - if possible - could provide elements that allow us to understand why it is so complicated for some people to understand physics science? The present work, which has mainly informative purposes, is a review of the literature on the subject, and as suggested in the title, it seeks to encourage reflection on the topic.
\end{abstract}

Keywords:

Physics thinking, scientific thinking, Physics education.

\section{Introducción}

Recientemente en agosto de 2019 se conmemoró el quince aniversario de la Licenciatura en Física y Tecnología Avanzada de la Universidad Autónoma del Estado de Hidalgo, en el marco de dichos festejos se presentó una conferencia con el título sugerente "Mientras más sabemos de mecánica cuántica, menos entendemos" en una parte de la exposición, el investigador que disertaba mencionó, palabras más palabras menos, que una persona no tiene que saber cómo funciona un automóvil para conducirlo, así como no se tiene que ser plomero para hacer uso de instalaciones hidráulicas, esto para contextualizar que en mecánica cuántica "hay cosas que sabemos que funcionan, y lo usamos, aunque no sepamos por qué funcionan".

Lo anterior fue motivo de reflexión para los autores, ya que en el caso del constructo teórico denominado

*Autor para la correspondencia: mcampos@uaeh.edu.mx

Correo electrónico: mcampos@ uaeh.edu.mx (Marcos Campos-Nava), aatr68@ hotmail.com (Agustín Alfredo Torres-Rodríguez) 
"Pensamiento matemático" o "Pensar matemáticamente" de lo cual se ha escrito e investigado a profundidad desde hace varias décadas, se considera que un rasgo de esta forma de pensar, es entender por qué las cosas funcionan de la forma en que funcionan en matemáticas.

Motivados por lo anterior, los autores se dieron a la tarea de reflexionar sobre el análogo al pensamiento matemático para los físicos ¿Qué significa pensar físicamente? ¿Cuándo una persona exhibe un pensamiento físico? ¿Qué características debería tener? Posteriormente a una profunda reflexión, surgió como proceso natural la iniciativa de realizar una revisión documental, para indagar el estado del arte referente a este tipo de pensamiento.

\section{El estado del arte sobre el pensamiento físico.}

En un intento por tratar de definir qué se debe entender por pensamiento físico, se puede recurrir en primera instancia al concepto de pensamiento científico, el cual es citado y caracterizado frecuentemente en la literatura; Zimmerman y Klahar, (2018) afirman que "El pensamiento científico ha mejorado la capacidad de los seres humanos para comprender, predecir y controlar las fuerzas naturales que dan forma a nuestro mundo.” (p.1).

En este orden de ideas, los mismos autores mencionan que el pensamiento científico comparte algunas características con otras formas de razonamiento, como por ejemplo, resolución de problemas; y que aunque en los niños de forma natural y espontánea surge la curiosidad por tratar de explicar ciertos fenómenos, el pensamiento científico debe desarrollarse por medio de la instrucción formal, de tal manera que el individuo que posea un pensamiento científico, tenga la capacidad de explorar el problema o fenómeno que quiere estudiar, pueda establecer hipótesis que lo intenten explicar, sea capaz de comprobar sus juicios por medio de la información que obtenga y en general capacidad de indagar, investigar y evaluar la evidencia obtenida. (Zimmerman y Klahar, 2018).

Sin embargo el constructo denominado pensamiento científico (scientific thinking), pese a ser citado numerosas veces en la literatura, tampoco está definido claramente, más bien, se enuncian algunas de sus características (competencias también a veces son llamadas), y en general se menciona lo que debiera ser capaz de hacer alguien que tiene desarrollado su pensamiento científico.

En este orden de ideas, Khun, (2011) establece que al querer definir el pensamiento científico, encuentra preferible definirlo con el término búsqueda del conocimiento (knowledge seeking), y además indica que existe diferencia entre pensar científicamente y entender científicamente.

Con base en lo anterior, antes de tratar de caracterizar el pensamiento físico, proponemos considerarlo como una forma de pensar, que emerge del pensamiento científico (búsqueda del conocimiento), que los seres humanos desarrollan de manera natural debido a su propia curiosidad, pero que si no es encauzado y acompañado de la instrucción académica formal, puede quedarse en un estadio sesgado en el cuál el individuo pudiera presentar esbozos de pensamiento científico, pero no es capaz de razonar científicamente.

En este contexto, Ossa-Cornejo, et al. (2018), afirman que:

De las habilidades necesarias para el razonamiento científico, destacan algunas como la indagación, el razonamiento y la argumentación, puesto que permiten determinar la validez de las fuentes de la información, evaluar las relaciones existentes entre los datos y comunicarlos de manera fundamentada. Estas habilidades están relacionadas con el pensamiento crítico y forman parte de lo que se conoce como alfabetización científica, definida por el Grupo de Expertos del área de Ciencias del PISA (Programme for International Student Assessment) como la capacidad de usar el conocimiento científico para identificar preguntas y para sacar hipótesis basadas en las pruebas, no solo con el fin de generar conocimiento, sino también para ayudar a tomar decisiones sobre el mundo natural y los cambios realizados en él a través de la actividad humana. (p.7)

En el caso particular de la Física, ésta se conceptualiza como una disciplina científica que se basa en la experimentación, es una ciencia factual, es decir, estudia los hechos de primera mano, los fenómenos naturales que el ser humano puede percibir por medio de los sentidos, y que son susceptibles de medirse de alguna forma, los objetos de estudio de la física no son abstracciones de la mente humana, como el caso de la lógica y/o la matemática (Gil, 2014; Halbwachs, 1976).

¿Cuál es el objeto de estudio de la física? probablemente si se le hace esta pregunta a un grupo de físicos de formación, la mayoría contestaría que la física estudia el universo y todo lo que hay en éste, desde los objetos macroscópicos como los sistemas solares, constelaciones y galaxias, hasta los objetos microscópicos como los átomos y las partículas fundamentales.

Indagando más a profundidad, y dado que con la definición anterior, se puede dilucidar que la biología y la química estudian objetos parecidos, probablemente los físicos explicarían que la física no es tan pretensiosa como la química y la biología al estudiar tan a fondo los componentes de la materia, y desentrañar los misterios de la vida, pero, que sin sus aportes, la química y la biología difícilmente podrían avanzar. (Alcubierre, 2005).

Por otro lado, decir que la física estudia objetos reales, es decir, que estudia el mundo real, lo que es medible, lo que se puede comprobar que existe, también presentaría un sesgo, pues la física no estudia como tal los objetos reales y 
tangibles; históricamente los físicos sí han tenido que realizar abstracciones por medio de modelos, para explicar los fenómenos de la naturaleza, y finalmente lo que el físico propone es una fotografía de la realidad, por lo regular explicado a través de modelos matemáticos. (Halbwachs, 1976).

En este orden de ideas, Espinoza (2015) en su recopilación del texto de Einstein e Infeld, afirma que:

\begin{abstract}
Los conceptos físicos son creaciones libres del espíritu humano y no están, por más que parezca, únicamente determinados por el mundo exterior. En nuestro empeño de concebir la realidad, nos parecemos a alguien que tratara de descubrir el mecanismo invisible de un reloj, del cual ve el movimiento de las agujas, oye el tic-tac, pero no le es posible abrir la caja que lo contiene. Si se trata de una persona ingeniosa e inteligente, podrá imaginar un mecanismo que sea capaz de producir todos los efectos observados: pero nunca estará segura de sí su imagen es la única que los pueda explicar. Jamás podrá compararla con el mecanismo real, y no puede concebir, siquiera, el significado de una comparación que le está vedada. (Espinoza, 2015, p. 53).
\end{abstract}

En concordancia con lo anterior, cuando se pretende estudiar un fenómeno físico, se trata de identificar qué conceptos físicos se relacionan con éste; los conceptos físicos sin embargo, no son una imagen fiel de la realidad del fenómeno, como ocurre con cualquier otro concepto definido por el ser humano, requiere un proceso de abstracción y hasta cierto punto de idealización, lo cual es común que ocurra en el estudio de la física; en otras palabras, plantear suposiciones razonables debería ser entonces un elemento que forme parte del pensamiento físico y probablemente sea un rasgo característico del razonamiento científico en general.

Sin pretender caer en lo meramente anecdótico, existe un conocido relato con intención de sátira y comicidad, que sin embrago pudiera aportar elementos para aclarar la diferencia entre algún rasgo del pensamiento físico respecto a otros tipos de pensamiento científico, por lo que a continuación se reproduce libremente una paráfrasis del mismo:

Un granjero requería de resolver el siguiente problema, necesitaba saber el volumen que ocupa una vaca, por lo que convoca a los profesionales que estén interesados en abordar el problema y proponer una solución satisfactoria. Acuden al llamado un ingeniero, un matemático y un físico; uno a uno expone su propuesta de solución al granjero.

Empieza el ingeniero, su propuesta consiste en construir un mecanismo con poleas que le permita levantar a la vaca por medio de un arnés y sumergirle en una enorme tina con agua, para que midiendo el volumen de líquido desplazado, obtenga el volumen de la vaca.
Continúa el matemático, quien propone parametrizar el volumen de la vaca, tomando algunas medidas de longitud básicas como la longitud de sus patas y el diámetro del cuello, realiza un programa computacional en el cual al introducir los parámetros solicitados, éste arroja un volumen estimado de cualquier vaca.

Toca el turno en tercer lugar al físico que empieza su propuesta diciendo "Supongamos que la vaca es esférica..."

El relato sin bien anecdótico, ejemplifica lo que se pudiera considerar un rasgo del pensamiento físico: la capacidad o la competencia de hacer suposiciones razonables que permitan simplificar los problemas para plantear un modelo matemático que se acerque a representarlo.

Recordemos que la rama más antigua de la física y la primera en abordarse formalmente -la mecánica clásicaprovee ejemplos significativos de éste rasgo del pensamiento físico, por ejemplo, la conocida ley de la caída libre de los cuerpos en la cinemática, resultado obtenido por Galileo Galilei, establece que el espacio recorrido por un cuerpo que cae libremente es directamente proporcional al cuadrado del tiempo transcurrido; sin embargo esto es cierto en el vacío, es decir en ausencia de fuerzas externas como la del rozamiento del aire. De manera natural es imposible tener una condición de vacío en nuestro planeta, esta debiera generarse artificialmente en todo caso, sin embargo la ley de la caída libre de Galileo, modela de forma adecuada muchos fenómenos que podemos observar cotidianamente, por ejemplo cuando una manzana cae de un árbol, o cuando se deja caer una piedra desde un puente.

En los cursos de física de secundaria y bachillerato, es común que aparezca como un tópico recurrente la ley de la caída libre, y se suelen resolver una gran cantidad de problemas que la involucran, además de realizar actividades experimentales que la corroboren, sin embargo, seguramente los estudiantes han experimentado dejar caer la pluma de un ave o una hoja de papel extendida, y habrán notado que su comportamiento no obedece a la ley de Galileo, tampoco lo obedece la envoltura de un cup cake o una bolsa de plástico si se dejan caer en cierta forma, ya que generan un efecto tipo paracaídas, de hecho la idea de los paracaídas es justamente provocar que un objeto en caída libre no obedezca a la ley de Galileo, o sería imposible practicar el paracaidismo.

Cabe mencionar que una implicación de la ley de la caída libre, es que un objeto que cae libremente en el vacío experimente un aumento constante de velocidad, es decir, conforme va cayendo su velocidad va aumentando, pero esto es en la condición del vacío; es bien sabido también, que las personas que practican el paracaidismo se dejan caer libremente desde una gran altura y durante varios segundos antes de abrir el paracaídas se pudieran considerar objetos en caída libre igual que la piedra que se deja caer de un puente y cuyo movimiento se puede predecir sin mayor dificultad 
con la ya citada ley; sin embargo, los paracaidistas experimentan luego de unos segundos de ir en caída libre, un movimiento prácticamente uniforme, es decir, su velocidad ya no aumenta aunque sigan en caída libre, de hecho su velocidad debido al rozamiento con el aire, no sobrepasa los 200 kilómetros por hora, (Ohanian y Markert, 2009, p.53) debido a eso es que el paracaídas es capaz de disminuir la velocidad al grado de permitirles llegar al suelo sin riesgos de accidentes.

Ejemplos como el anterior sobran, muchas de las leyes de la física que se estudian en la escuela, requieren de aceptar ciertas restricciones o como en el relato de la vaca, hacer suposiciones que sean razonables, por ejemplo que las moléculas de un gas son como pelotas de billar y que al chocar entre ellas no se deforman, o suponer que la corriente eléctrica viajando a través de un conductor metálico como el cobre es análogo al agua que fluye por una tubería, lo cual, evidentemente en la realidad no es así, pero en algunos casos, permite plantear modelos matemáticos que explican bajo esas condiciones o suposiciones, al fenómeno físico en estudio.

Retomando el relato de la vaca, un rasgo que se podría considerar distintivo entre la forma de resolver problemas de un ingeniero versus la forma en la que los resuelve un físico, es la practicidad, es decir, el ingeniero busca resolver el problema de la forma más simple, avalado por algún principio físico y/o matemático que aprendió en la escuela (y muchas otras veces seguramente apoyado en su experiencia), el físico en cambio teoriza, hace suposiciones razonables, plantea hipótesis, trata de establecer modelos teóricos; por otro lado, el ingeniero resuelve problemas con lo que tiene a su alcance. Es curioso sin embargo, hacer esta distinción, cuando para el imaginario colectivo, un ingeniero es un profesionista que estudió mucho de física y de matemáticas y lo aplica para la solución de problemas tecnológicos; de hecho, históricamente en México, los primeros profesores de física en las universidades no fueron físicos de formación sino ingenieros, y las primeras escuelas, facultades o institutos donde se ofertaron inicialmente las carreras de física y se hicieron las primeras investigaciones relacionadas a esta disciplina, también tuvieron como figuras centrales a ingenieros mexicanos (Ramos-Lara, 2015; Tanamachi-Castro y Ramos-Lara, 2015, Ramos-Lara, 2007).

\section{Algunos ejemplos de ausencia de pensamiento físico entre estudiantes.}

En un primer curso de física a nivel universitario, es común que se planteen problemas de cinemática sobre caída libre entre otros diversos temas de mecánica. Por medio de la experiencia recopilada tras años de haber atendido a distintos grupos y tras platicar con otros colegas profesores, se ha identificado que en cada generación, hay alumnos que en aquellos problemas en los cuáles se suelen indicar que un objeto se deja caer desde el reposo a cierta altura, son capaces de identificar que se trata de movimiento rectilíneo uniformemente acelerado, también tienen a su disposición los modelos matemáticos que describen dicho fenómeno, pero suelen preguntar ¿qué hago si no tengo la velocidad inicial y la aceleración? Esto pudiera ser un rasgo distintivo de que a ese tipo de estudiantes, les cuesta trabajo hacer suposiciones razonables, entrever en la información datos adicionales como las condiciones iniciales.

También a lo largo de la experiencia, se ha podido identificar que cuando los problemas describen cierto fenómeno asociado al movimiento de un objeto, por ejemplo aquellos problemas que describen verbalmente los desplazamientos de una persona y luego solicitan a partir de la información, realizar una representación gráfica (por ejemplo la gráfica de distancia versus tiempo de alguien que salió de su casa, fue a la tienda que está a dos cuadras, regresó a su casa por más dinero y luego fue a otra tienda situada del otro lado y regresó a su casa, etcétera) suele resultarles complicado para algunos estudiantes y muy claro para otros, lo cual pudiera ser otro rasgo distintivo del pensar físicamente, ya que como una ciencia experimental, una competencia fundamental es poder expresar información de fenómenos en forma gráfica.

Aunado a lo anterior, otro tipo de problemas que parecieran no dar datos o dar pocos y solicitan generalizaciones, como el que menciona que se deja caer una pelota desde cierta altura, la cual llega al suelo y permanece en contacto con éste por un periodo breve de tiempo antes de rebotar, y a partir de esto se solicita el valor de la desaceleración que sufrió dicha pelota, resulta para la mayoría de estudiantes novatos, un problema complicado, pues no conciben por ejemplo que la desaceleración se puede expresar en términos de "g" o no consideran viable suponer que si fue una persona la que dejó caer la pelota, esta debió ser soltada aproximadamente de una altura de un metro y medio.

Otro caso muy conocido de asunción razonable que se hace en física, es la de suponer que un objeto material puede ser considerado una partícula, es frecuente que además esta partícula sea el centro de masa; en problemas de mecánica típicos de los libros de texto, los estudiantes consideran que bloques, cajas, automóviles, personas, etcétera, pueden ser idealizados como una partícula pero con masa (masa puntual); cuando se interroga a los estudiantes de ¿por qué esta suposición? ¿Por qué no importa la forma o las dimensiones para el análisis? Difícilmente son capaces alguno de dar una razón acertada, simplemente ni siquiera se cuestionan porqué se puede suponer esta idealización para abordar problemas de física.

Los ejemplos anteriores invitan a considerar que parte de pensar físicamente es tener la capacidad de, a partir de la observación de un fenómeno natural, poder matematizar (encontrar un modelo matemático que lo pueda describir) y viceversa, teniendo un modelo matemático que de antemano se sabe describe un fenómeno natural, darle sentido en términos de las condiciones físicas inherentes al fenómeno. 


\section{Posibles indicadores para caracterizar el pensamiento físico.}

Recapitulando, el pensamiento físico tiene rasgos en común con otras formas de pensamiento o de razonar, por ejemplo, con la resolución de problemas en matemáticas, o con el pensamiento científico y el pensamiento crítico, sin embargo, tiene también sus particularidades, lo que lo hace distintivo de otras formas de pensar, un perfil que no surge de manera espontánea sino que se va construyendo progresivamente en buena medida por la formación académica a la que es expuesto un estudiante durante su trayecto escolar, es de suponer, que inclusive el perfil de los profesores de física, tenga implicación en el desarrollo del pensamiento físico de los estudiantes, y por consecuencia en el de los futuros físicos; resulta plausible proponer que en la medida en la que un estudiante de bachillerato tenga más desarrollado el pensamiento físico, tendrá mejor oportunidad de emprender y desarrollar adecuadamente una carrera en ciencias físicas.

Cabe señalar que el pensamiento científico está estrechamente relacionado al pensamiento crítico, y por ende al pensamiento físico y aunque estos constructos se han investigado y definido desde hace décadas, como mencionan Loaiza y Delia, (2018):

\section{[...] definir el pensamiento crítico, sus habilidades y sus formas de aplicación, no es tarea fácil; de allí el auge investigativo que ha generado en las últimas décadas, convirtiéndolo en objeto de estudio. En estas investigaciones se observan distintas rutas que intentan conceptualizar, describir, aplicar y desarrollar el pensamiento crítico en el aula, abordándolo desde diferentes disciplinas del saber y en contextos diversos, que determinan la importancia del pensamiento crítico en el desarrollo de la vida académica y personal de los educandos, y hacen visible la necesidad de implementar estrategias de enseñanza de habilidades cognitivas, metacognitivas y disposicionales en cualquier nivel de educación. (p.4).}

Empero resulta escasa la información que se puede encontrar en la literatura respecto a una definición formal de lo que podemos entender como pensamiento físico, autores como Aragón y Marín (2010), definen lo que han llamado pensamiento físico-matemático de la siguiente manera:

El que una persona consiga construir un concepto, una idea, o una explicación pertinente sobre alguna fenomenología física, depende significativamente del nivel de desarrollo de su pensamiento, caso contrario a si consideramos que el hecho de enseñar física forma automáticamente en nuestros estudiantes dicho pensamiento, por lo general el aprendizaje de algún concepto involucra que de antemano el estudiante haya desarrollado algunas habilidades que le permitan: comparar, describir, analizar, sintetizar, abstraer, modelar, etc. A este conjunto de habilidades le hemos denominado Pensamiento Físico-Matemático (PFM). (p.2)

¿Cómo se puede caracterizar el nivel o grado de desarrollo de pensamiento físico de un estudiante egresado de bachillerato?

La respuesta a la pregunta anterior por supuesto no es trivial ni única, de hecho tras la revisión de la literatura, algo que queda claro debido a la escasez de fuentes que puntualmente hablen al respecto, es que está en construcción, y este trabajo pretende sumar a esos esfuerzos, en una primera aproximación se puede partir de lo que está declarado en documentos oficiales sobre el perfil progresivo de los estudiantes de bachillerato en México, particularmente el primer curso de física debería desarrollar algunas competencias que son compatibles con el discurso que hasta ahora se ha manejado sobre el pensamiento y el razonamiento científico. En este orden de ideas, se presentan a continuación algunas competencias declaradas en los programas de estudio de bachillerato del área de física:

$>$ Identifica problemas, plantea preguntas de carácter científico y formula hipótesis para responderlas.

> Contrasta los resultados obtenidos de una investigación o experimento con hipótesis previas.

> Hace explícitas las nociones científicas que sustentan los procesos para la solución de problemas cotidianos. (SEP, 2018, p. 10, extracto programa de estudios de Física I).

Plantear preguntas acerca de los fenómenos físicos que el estudiante puede observar en su cotidianeidad, proponer respuestas provisionales con base en sus conocimientos previos y ser capaz de evaluar si sus hipótesis son o no plausibles, debieran ser indicadores de lo que podría llamarse la competencia del pensamiento físico. (OssaCornejo et al, 2018; Chamizo e Izquierdo, 2007).

Una persona con pensamiento crítico se caracteriza por saber formular problemas y preguntas vitales, tener claridad y precisión respecto a la información, acumular y evaluar información relevante, usar ideas abstractas para interpretar esa información efectivamente, llegar a conclusiones y soluciones, probándolas con criterios y estándares relevantes; además de pensar con una mente abierta dentro de los sistemas alternos de pensamiento, reconocer y evaluar según sea necesario, los supuestos, implicaciones y consecuencias prácticas, y finalmente, idear soluciones a problemas complejos comunicándose certera $y$ efectivamente. (Ossa-Cornejo et al., 2018, p. 5). 


\section{Reflexiones Finales}

La configuración de un marco de referencia resulta una clave necesaria para poder caracterizar el pensamiento físico. Este tipo de pensamiento comúnmente se relaciona con el pensamiento matemático, el pensamiento (método) científico y la resolución de problemas.

El concepto del pensamiento crítico es también un referente indispensable para definir el pensamiento físico, dado que ambos están estrechamente imbricados.

La relevancia de estos tipos de pensamiento y su relación con la física, se comprende si consderamos por ejemplo en que el pensar físicamente, requiere de la capacidad de ir desde la observación de un fenómeno natural, hasta arribar a un modelo matemático que lo pueda describir, y también en sentido inverso, teniendo un modelo matemático que describe un fenómeno natural, darle sentido o significado físicos.

Es importante reconocer que en la actualidad la física, en su intento por comprender la naturaleza de los distintos fenómenos que estudia, va construyendo un corpus teórico cada vez más robusto, y para ello le resulta indispensable que los futuros físicos, esto es, los actuales estudiantes de física logren desarrollar elementos del pensamiento científico y crítico en lo general, así como del pensamiento matemático, en particular, para ir configurando un pensamiento propio, que a su vez se vaya constituyendo en un constructo que se pueda identificar plenamente con el nombre de pensamiento físico.

\section{Agradecimientos}

Los autores agradecen al Doctor Roberto Noriega Papaqui por las ideas y ejemplos que tuvo a bien proporcionar para la realización de este trabajo.

\section{Referencias}

Alcubierre, M. (2005). Soy Físico. ¿Cómo ves? No. 78, 1014.

Aragón, P. y Marín, C. (2010). El pensamiento físicomatemático como un objeto de estudio de la didáctica de la física. En memorias del Congreso Iberoamericano de Educación METAS 2021. Buenos Aires, Argentina.

Chamizo, J. A., \& Izquierdo, M. (2007). Evaluación de las competencias de pensamiento científico. Alambique: Didáctica de las ciencias experimentales (51), 9-19.

Espinoza, J. (2015). Eureka enseñanza de las ciencias físicas. Recuperado el 25 de Junio de 2019, de http://www.umce.cl/joomlatools-files/docmanfiles/universidad/revistas/eureka/revistaeureka_05_07-
Leyendo-a_La-Fisica-aventura-del-pensamiento-deAlbert.pdf

Gil, S. (2014). Experimentos de Física usando las TIC y elementos de bajo costo. Editorial Alfaomega: México.

Halbwachs, F. (1976). El pensamiento físico del niño y el sabio. Marfil, Alcoy.

Khun, D. (2011). What is Scientific Thinking and How Does it Develop? In U. Goswami (Ed.), Handbook of Childhood Cognitive Development (Blackwell): New York.

Ohanian, H y Markert, J. (2009). Física para Ingeniería y Ciencias. Ed McGraw Hill: México.

Ossa-Cornejo, C., Palma-Luengo, M., Lagos-San Martín, N., y Díaz-Larenas, C. (2018). Evaluación del pensamiento crítico y científico en estudiantes de pedagogía de una universidad chilena. Revista Electrónica $\quad$ Educare, $\quad 22(2), \quad 1-18$. doi:http://dx.doi.org/10.15359/ree.22-2.12.

Ramos-Lara, M. (2015). Figuras y entidades pioneras de la física en México. Revista Mexicana de Física E (61), 93103.

Ramos-Lara, M. (2007). Los ingenieros promotores de la física académica en México (1910-1935). Revista Mexicana de Investigación Educativa, 12(35), 12411265.

SEP. (2018). SECRETARÍA DE EDUCACIÓN PÚBLICA. Recuperado el 27 de Junio de 2019, de https://www.dgb.sep.gob.mx/informacionacademica/programas-de-estudio.php

Smith, A. (1936). Physics as a Way of Thinking. Law Journal, 241-259.

Tanamachi-Castro, G., y Ramos-Lara, M. (2015). La escuela nacional de ingenieros y las ciencias físicas en los albores del siglo XX. Revista Mexicana de Investigación Educativa, 20(65), 557-580.

Zimmerman, C., y Klahar, D. (2018). Development of scientific Thinking. En J. T. Wixted (Ed.), Handbook of Experimental Psycology and Cognitive Neurosciences (Fourth ed., págs. 1-25). John Wiley \& Sons. doi:10.1002/9781119170174.epcn407

\section{Apéndice A. Mapa conceptual sobre el pensamiento físico.}

El pensamiento físico en los seres humanos se desarrolla desde edad temprana con la intención de explicar los fenómenos naturales de su entorno, por ejemplo la caída de un objeto, el ciclo del día y la noche, la diferencia entre sustancias sólidas como una piedra y líquidos como el agua. El pensamiento físico se basa en la observación y experimentación, en otras palabras es un razonamiento inductivo, que permite contrastar hipótesis y preconceptos, con la finalidad de crear abstracciones mentales que puedan representarse como relaciones matemáticas, con la finalidad de evitar conclusiones erróneas. Este pensamiento está relacionado con el denominado pensamiento científico, que es parte del constructo anglosajón denominado Scientific Literacy (alfabetización científica), indispensable para vivir en una sociedad moderna que permita comprender, 
investigar e interpretar contenidos científicos actuales como el cambio climático, la evaluación de tratamientos médicos más efectivos entre otros. La importancia del constructo denominado pensamiento científico radica en la influencia que tiene en el desarrollo del currículo de ciencias, este concepto surgió originalmente cuando Albert Einstein sugirió a Jean Piaget indagar sobre el pensamiento científico. (Smith, 1936; Zimmerman Y Klahar, 2018).

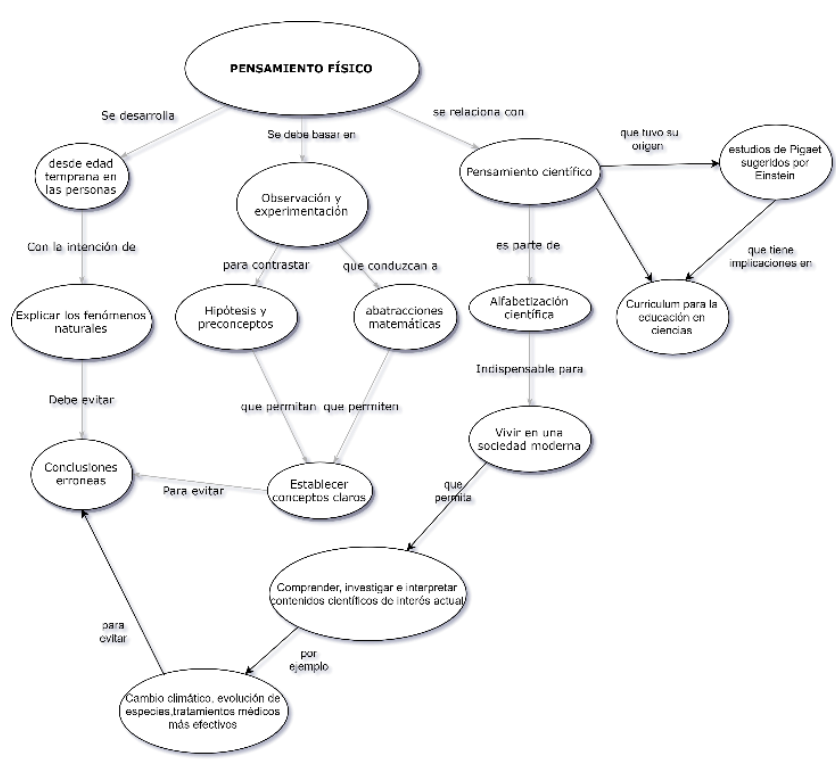

